\title{
新型脱氢枞胺衍生物的合成及其抗自由基活性
}

\author{
陆 洲刘超祥余星林中祥*
}

(南京林业大学化学工程学院＼cjkstart南京 210037)

\begin{abstract}
摘要 通过不同的方法在脱氢枞胺中引入酰腙、没食子酸、肜、异烟基等对清除自由基有效果的基团, 设计合成了几 种新型的脱氢枞胺衍生物. 利用 ${ }^{1} \mathrm{H} N M R,{ }^{13} \mathrm{C} N M R$, IR 和 HRMS 对所有合成的化合物进行了结构表征. 测试了所合成 的化合物对清除超氧阴离子 $\left(\mathrm{O}_{2}^{-}\right)$和二苯代苦味酰基自由基( $\mathrm{DPPH} \bullet$ 的活性，其中 $N-(3,4,5$-三羊基苯甲酰基)-脱氢枞胺 (6) 对 $\mathrm{O}_{2}^{-}$的抑制率达到 $38.18 \%$, 是常用抗氧化药物 $\mathrm{Vc}(18.35 \%)$ 的两倍以上; 对 $(\mathrm{DPPH} \bullet)$ 的半数抑制浓度为 $0.002 \times 10^{3}$ $\mathrm{mg} / \mathrm{L}$, 远优于 $\mathrm{Vc}\left(0.236 \times 10^{3} \mathrm{mg} / \mathrm{L}\right)$.
\end{abstract}

关键词＼cjkstart脱氢枞胺; 抗自由基; 酰腙; 没食子酸

\section{Synthesis and Anti-free Radical Activities of Several Novel Derivatives of Dehydroabietylamine}

\author{
Lu, Zhou Liu, Chaoxiang Yu, Xing Lin, Zhongxiang* \\ (College of Chemical Engineering Nanjing Forestry University, Nanjing 210037)
}

\begin{abstract}
A series of novel derivatives of dehydroabietylamine were synthesized by introducing some groups with good free radical scavenging activities into the dehydroabietylamine, such as hydrazone, gallic acid, oxime and isoniazid. Structures of the synthesized compounds were characterized by IR, ${ }^{1} \mathrm{H}$ NMR, ${ }^{13} \mathrm{C}$ NMR, MS and HRMS techniques. All the compounds were tested the ability on scavenging superoxide anion radical $\left(\mathrm{O}_{2}^{-}\right)$and 1,1-dipheny-2-picrylhydrazyl (DPPH•). The results indicate that the inhibitory rate of $\mathrm{O}^{2-}$ by (6) $(38.18 \%)$ is twice as much as that of $\mathrm{Vc}(18.35 \%)$, and its capacity on scavenging $\mathrm{DPPH} \bullet\left(\mathrm{IC}_{50}=0.002 \times 10^{3} \mathrm{mg} / \mathrm{L}\right)$ is much better than $\mathrm{Vc}\left(\mathrm{IC}_{50}=0.236 \times 10^{3} \mathrm{mg} / \mathrm{L}\right.$.
\end{abstract}

Keywords dehydroabietylamine; anti-free radical; acylhydrazone; gallic acid

自由基是一类具有高度活性的物质，研究表明它参 与了动脉湅样硬化、阿尔茨海默病、高血压等多种疾病 的发生 ${ }^{[1 \sim 2]}$, 而自由基清除剂能够清除自由基, 保护机 体免受氧化损害, 研究开发新的抗自由基药物成为当今 最热门的课题之一. 脱氢枞胺是一种具有三环菲结构的 松香改性产品, 它是歧化松香胺的主要成分. 近年来越 来越多的学者对脱氢枞胺及其衍生物的抗菌、杀虫、抗 病毒、抗肿瘤活性进行研究 ${ }^{[3 \sim 10]}$, 这些研究表明脱氢枞 胺在药物研究和生物学等方面正显示出越来越重要的 作用. 但是, 一直以来脱氢枞胺的抗自由基作用没有见 文献报道, 本工作通过连苯三酚法 ${ }^{[1]}$ 和 DPPH 法 $^{[12]}$ 测试 了脱氢枞胺清除超氧阴离子 $\left(\mathrm{O}_{2}^{-}\right)$和二苯代苦味酰基自 由基( $\mathrm{DPPH} \bullet$ 的能力, 测试结果表明脱氢枞胺虽然有抗
自由基能力，但和常用抗自由基药物 $\mathrm{Vc}^{[13]}$ 相比并没有 优势. 为了提高其抗自由基效果，本工作采用不同的方 法在脱氢枞胺中引入酰腙、没食子酸、肜基、硫艮、异 烟基等有较好抗自由基活性的基团 ${ }^{[14 ~ 18]}$, 合成了几种 结构新颖的脱氢枞胺衍生物(Scheme 1, Eq. 1), 并测试 了它们对自由基的清除作用. 结果显示部分化合物清除 自由基的效果远优于 Vc. 希望这些研究能为后续开发 脱氢枞胺类抗氧化药物提供参考.

\section{1 实验部分}

\section{1 试剂与仪器}

脱氢枞胺, 用桂林松泉林化工业有限责任公司生产

\footnotetext{
*E-mail: linzhongxiang36@gmail.com

Received November 9, 2012; revised December 6, 2012; published online December 10, 2012.

Project supported by the National Natural Science Foundation of China (No. 31170536) and the Priority Academic Program Development of Jiangsu Higher Education Institutions.

国家自然科学基金(No. 31170536)和江苏高校优势学科建设工程资助项目.
} 


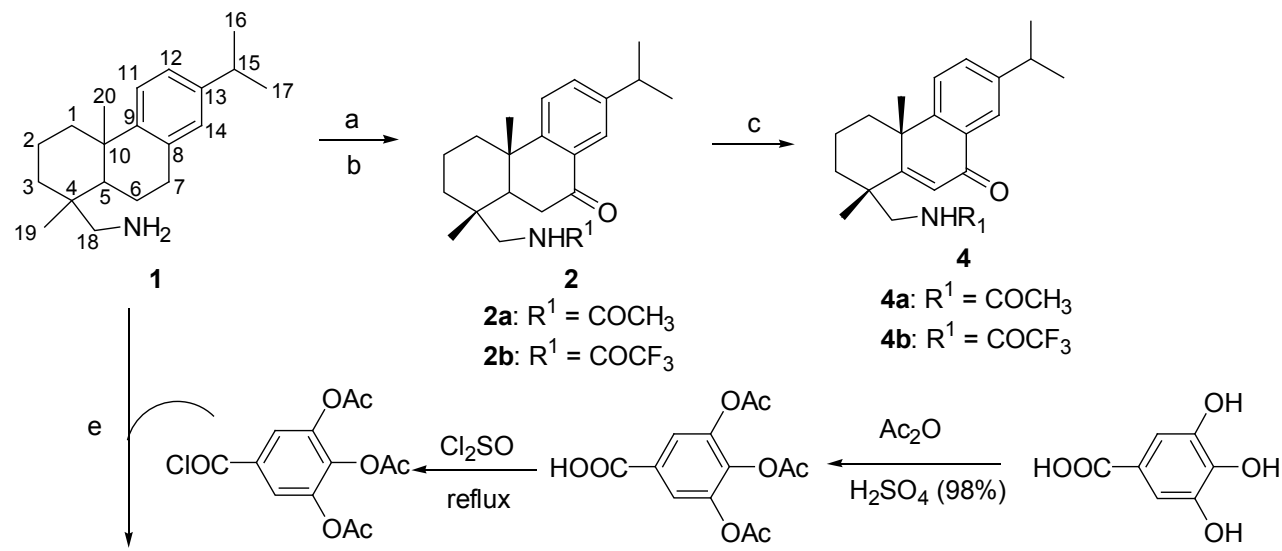

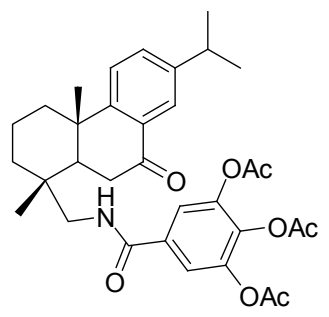

5

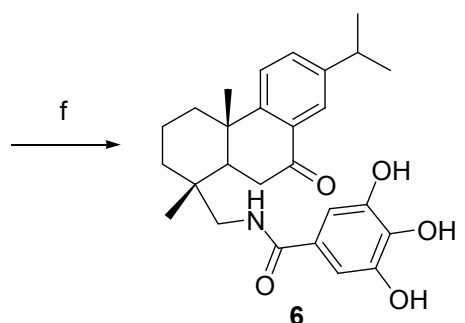

Reagents and conditions: (a) $(\mathrm{AcO})_{2} \mathrm{O}$ or $\left(\mathrm{CF}_{3} \mathrm{CO}\right)_{2} \mathrm{O}, 10 \mathrm{~h}, 30^{\circ} \mathrm{C}(87 \%)$; (b) $\mathrm{CrO}_{3}, \mathrm{CH}_{3} \mathrm{COOH}, 3 \mathrm{~h}, 0 \sim 5{ }^{\circ} \mathrm{C}(76 \%)$; (c) $\mathrm{SeO}_{2}, \mathrm{CH}_{3} \mathrm{COOH}$, reflux (50\%); (e) $\mathrm{CH}_{2} \mathrm{Cl}_{2}$, TEA, $4 \mathrm{~h}, 30^{\circ} \mathrm{C}(69 \%)$; (f) $\mathrm{HHA}$ (Hydrazine hydrate) $-\mathrm{CH}_{2} \mathrm{Cl}_{2}, \mathrm{CH}_{3} \mathrm{CH} \mathrm{OH}_{2} \mathrm{O} 2{ }^{\circ} \mathrm{C}, 6 \mathrm{~h}(98 \%)$

Scheme 1

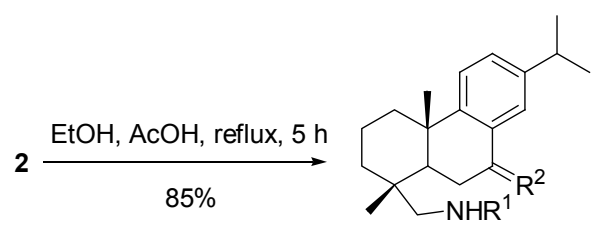

3

3a: $\mathrm{R}^{1}=\mathrm{COCH}_{3}, \mathrm{R}^{2}=\mathrm{NOCH}_{3}$

3b: $\mathrm{R}^{1}=\mathrm{COCH}_{3}, \mathrm{R}^{2}=\mathrm{NOCH}_{2}$

3c: $\mathrm{R}^{1}=\mathrm{COCH}_{3}, \mathrm{R}^{2}=\mathrm{NNHC}(\mathrm{O}) \mathrm{NH}_{2}$

3d: $\mathrm{R}^{1}=\mathrm{COCH}_{3}, \mathrm{R}^{2}=\mathrm{NNHC}(\mathrm{S}) \mathrm{NH}_{2}$

3e: $\mathrm{R}^{1}=\mathrm{COCH}_{3}, \mathrm{R}^{2}=\mathrm{NNHC}(\mathrm{O})$

3f: $\mathrm{R}^{1}=\mathrm{COCF}_{3}, \mathrm{R}^{2}=\mathrm{NOH}$

3g: $\mathrm{R}^{1}=\mathrm{COCF}_{3}, \mathrm{R}^{2}=\mathrm{NNHC}(\mathrm{O}) \mathrm{NH}_{2}$

3h: $\mathrm{R}^{1}=\mathrm{COCF}_{3}, \mathrm{R}^{2}=\mathrm{NNHC}(\mathrm{O})$

的歧化松香胺按文献[19]提纯得到; 其它化学试剂为分 析纯.

美国 Nicolet 360 傅立叶红外光谱仪( $\mathrm{KBr}$ 压片); Bruker-AVANCE AV500 核磁共振波谱仪 $\left(\mathrm{CDCl}_{3}\right.$ 为溶 剂, TMS 为内标); BIFLEX III 型 MALDI-TOF 质谱仪; XT6 显微熔点测定仪 (上海荆和分析仪器有限公司); TU-1900 双光束紫外可见分光光度计(北京普析通用仪 器有限责任公司). 柱层析采用柱层析硅胶 $(200 \sim 300$
目)(青岛海洋化工厂生产).

\section{2 合成}

\subsection{1 化合物 $\mathbf{2 a}, \mathbf{2 b}$ 的合成}

中间产物乙酰脱氢枞胺-7-酮(2a) 和三氟乙酰脱氢 枞胺-7-酮(2b)的合成可以参考文献[20], 分两步合成.

\subsection{2 化合物 $\mathbf{3 a} \sim \mathbf{3 h}$ 的合成}

以目标化合物 $3 \mathbf{a}$ 的合成为例，将 $8.79 \mathrm{mmol} \mathbf{2 a}$ 用 $50 \mathrm{~mL}$ 乙醇溶解, 加入 $150 \mathrm{~mL}$ 四口瓶中, 再加入 8.8 $\mathrm{mmol}$ 甲氧胺盐酸盐和少量醋酸, 加热回流 $8 \mathrm{~h}$. 反应结 束后减压蒸除溶剂, 残留物用硅胶柱层析[洗脱剂: $V($ 石 油醚) $: V$ (乙酸乙酯 $)=5: 5$ ]分离得到目标产物 3a. 用 类似的方法得到产物 $\mathbf{3 b} \sim \mathbf{3 h}$.

乙酰脱氢枞胺-7-( $O$-甲基肟)(3a): 淡黄色固体，产 率 $85 \%$. m.p. $88.5 \sim 89.7{ }^{\circ} \mathrm{C} ;{ }^{1} \mathrm{H}$ NMR $\left(500 \mathrm{MHz}, \mathrm{CDCl}_{3}\right.$ ) $\delta: 7.76(\mathrm{~s}, 1 \mathrm{H}, \mathrm{ArH}), 7.17$ (d, $J=12.2 \mathrm{~Hz}, 2 \mathrm{H}, \mathrm{ArH}), 5.48$ (s, 1H, NH), $4.11 \sim 3.92\left(\mathrm{~s}, 3 \mathrm{H}, \mathrm{OCH}_{3}\right), 3.31(\mathrm{dt}, J=18.9$, $9.5 \mathrm{~Hz}, 9.5 \mathrm{~Hz}, 1 \mathrm{H}), 2.98(\mathrm{~s}, 1 \mathrm{H}), 2.88 \sim 2.80(\mathrm{~s}, 1 \mathrm{H})$, $2.25 \sim 2.56(\mathrm{~m}, 2 \mathrm{H}), 1.95(\mathrm{~s}, 3 \mathrm{H}), 1.78 \sim 1.71(\mathrm{~m}, 2 \mathrm{H})$, $1.51(\mathrm{dd}, \quad J=13.5,5.0 \mathrm{~Hz}, 2 \mathrm{H}), 1.33 \sim 1.17(\mathrm{~m}, 9 \mathrm{H})$, $1.15 \sim 1.00(\mathrm{~m}, 6 \mathrm{H}) ;{ }^{13} \mathrm{C} \mathrm{NMR}\left(125 \mathrm{MHz}, \mathrm{CDCl}_{3}\right) \delta$ : $170.41(\mathrm{C}=\mathrm{O}), 154.25(\mathrm{C}=\mathrm{N}), 148.78(\mathrm{C}-9), 146.46$ (C-13), 128.87 (C-12), 127.64 (C-8), 122.89 (C-14), 122.29 (C-11), $61.98\left(\mathrm{OCH}_{3}\right), 49.39,42.08,37.50,37.46$, $36.87,36.12,33.65,24.14,23.66,23.46,23.03,22.34$, 
18.55, 18.14; IR (KBr) v: 3302, 2959, 2930, 2869, 1721, 1651, 1460, 1378, 1052, 889, $755 \mathrm{~cm}^{-1}$; ESI-MS (+) $\mathrm{m} / \mathrm{z}$ : $371.2[\mathrm{M}+\mathrm{H}]^{+}, 393.1[\mathrm{M}+\mathrm{Na}]^{+}$; HRMS (EI) calcd for $\mathrm{C}_{23} \mathrm{H}_{34} \mathrm{~N}_{2} \mathrm{O}_{2}$ 371.2699, found 371.2702.

乙酰脱氢枞胺-7-( $O$-芐基肟)(3b): 淡黄色粘稠液体, 产率 87\%. ${ }^{1} \mathrm{H}$ NMR $\left(500 \mathrm{MHz}, \mathrm{CDCl}_{3}\right) \delta: 7.76(\mathrm{~s}, 1 \mathrm{H}$, ArH), 7.44 (d, $J=7.0 \mathrm{~Hz}, 2 \mathrm{H}, \operatorname{ArH}), 7.37$ (dd, $J=7.4,1.9$ $\mathrm{Hz}, 2 \mathrm{H}, \mathrm{ArH}), 7.29$ (s, 1H, ArH), 7.18 (d, J=4.1 Hz, 2H, ArH), 5.45 (s, 1H, NH), 5.27 (d, J=5.2 Hz, 2H, NO-CH ${ }_{2}$, $3.31(\mathrm{dd}, J=13.8,7.8 \mathrm{~Hz}, 7.8 \mathrm{~Hz}, 1 \mathrm{H}), 2.97 \sim 2.86(\mathrm{~m}$, $3 \mathrm{H}), 2.59(\mathrm{~s}, 1 \mathrm{H}), 1.90(\mathrm{~s}, 3 \mathrm{H}), 1.75 \sim 1.67(\mathrm{~m}, 2 \mathrm{H}), 1.51$ $(\mathrm{dd}, J=13.5,5.0 \mathrm{~Hz}, 2 \mathrm{H}), 1.47 \sim 1.42(\mathrm{~m}, 1 \mathrm{H}), 1.27 \sim 1.23$ $(\mathrm{m}, 8 \mathrm{H}), 1.09$ (s, 3H), $1.03(\mathrm{~s}, 3 \mathrm{H}) ;{ }^{13} \mathrm{C} \mathrm{NMR}(125 \mathrm{MHz}$, $\left.\mathrm{CDCl}_{3}\right) \delta: 170.26(\mathrm{C}=\mathrm{O}), 154.58(\mathrm{C}=\mathrm{N}), 148.81,146.36$, $138.21,128.93,128.42,128.33,128.29,128.24,127.69$, 127.67, 122.87, 122.40, $76.31\left(\mathrm{NO}-\mathrm{CH}_{2}\right), 49.37,42.23$, $37.51,37.46,36.88,36.10,33.60,24.06,23.67,23.41$, 23.06, 22.54, 18.56, 18.13, 14.14; IR (KBr) v: 3304, 2958, 2927, 2868, 1737, 1652, 1558, 1456, 1367, 1287, 1018, 940, $739 \mathrm{~cm}^{-1}$; ESI-MS (+) $m / z: 447.3[\mathrm{M}+\mathrm{H}]^{+}$; HRMS (EI) calcd for $\mathrm{C}_{29} \mathrm{H}_{38} \mathrm{~N}_{2} \mathrm{O}_{2} 447.3016$, found 447.3012.

乙酰脱氢枞胺-7-氨基嫝酰腙(3c): 白色粉末, 产率 86\%, m.p.133.3 134.5 ${ }^{\circ} \mathrm{C} ;{ }^{1} \mathrm{H}$ NMR (500 MHz, $\mathrm{CDCl}_{3}$ ) $\delta: 10.40(\mathrm{~s}, 1 \mathrm{H}, \mathrm{NNHC}=\mathrm{O}), 7.79(\mathrm{~s}, 1 \mathrm{H}, \mathrm{ArH}), 7.21(\mathrm{~s}$, 2H, ArH), 6.33 (s, 2H, NH $), 5.93$ (s, 1H, NH), 3.93 (dd, $J=13.2,8.3 \mathrm{~Hz}, 1 \mathrm{H}), 3.07 \sim 2.87(\mathrm{~m}, 2 \mathrm{H}), 2.31 \sim 2.79(\mathrm{~m}$, $3 \mathrm{H}), 2.04(\mathrm{~d}, J=12.9 \mathrm{~Hz}, 3 \mathrm{H}), 1.81(\mathrm{~s}, 3 \mathrm{H}), 1.67 \sim 1.54$ $(\mathrm{m}, 3 \mathrm{H}), 1.52 \sim 1.29(\mathrm{~m}, 6 \mathrm{H}), 1.12(\mathrm{~d}, J=4.2 \mathrm{~Hz}, 6 \mathrm{H}) ;{ }^{13} \mathrm{C}$ NMR (125 MHz, $\left.\mathrm{CDCl}_{3}\right) \delta: 170.49\left(\mathrm{COCH}_{3}\right), 159.04$ $\left(\mathrm{CONH}_{2}\right), 148.77(\mathrm{C}=\mathrm{N}), 146.39,146.09,130.96,127.33$, 122.73, 122.38, 51.04, 44.45, 37.71, 37.50, 36.80, 36.65, $33.71,24.77,24.07,23.81,23.55,23.06,18.24,17.41$; IR (KBr) v: 3483, 3320, 2959, 2928, 1692, 1572, 1420, 1384, 1127, $550 \mathrm{~cm}^{-1}$; ESI-MS (+) $m / z: 399.2[\mathrm{M}+\mathrm{H}]^{+}, 421.2$ $[\mathrm{M}+\mathrm{Na}]^{+}$; HRMS (EI) calcd for $\mathrm{C}_{23} \mathrm{H}_{34} \mathrm{~N}_{4} \mathrm{O}_{2}$ 399.2764, found 399.2760 .

乙酰脱氢枞胺-7-硫代氨基脲酰腙(3d): 淡黄色粉 末, 产率 88\%. m.p. $145.6 \sim 146.8{ }^{\circ} \mathrm{C} ;{ }^{1} \mathrm{H}$ NMR (500 $\left.\mathrm{MHz}, \mathrm{CDCl}_{3}\right) \delta: 9.58(\mathrm{~s}, 1 \mathrm{H}, \mathrm{NNHC}=\mathrm{S}), 7.75(\mathrm{~s}, 1 \mathrm{H}$, ArH), 7.45 (s, 1H, $\mathrm{NH}_{2}$ ), 7.22 (dd, $J=6.9,5.0 \mathrm{~Hz}, 2 \mathrm{H}$, ArH), $6.80\left(\mathrm{~s}, 1 \mathrm{H}, \mathrm{NH}_{2}\right), 5.77(\mathrm{~s}, 1 \mathrm{H}, \mathrm{NH}), 3.30 \sim 3.13(\mathrm{~m}$, $2 \mathrm{H}), 3.05 \sim 2.94(\mathrm{~m}, 2 \mathrm{H}), 2.52(\mathrm{~s}, 1 \mathrm{H}), 2.28(\mathrm{~s}, 1 \mathrm{H}), 1.92$ $(\mathrm{s}, 3 \mathrm{H}), 1.78 \sim 1.69(\mathrm{~m}, 2 \mathrm{H}), 1.48 \sim 1.64(\mathrm{~m}, 3 \mathrm{H}), 1.41 \sim$ $1.29(\mathrm{~m}, 1 \mathrm{H}), 1.28 \sim 1.26(\mathrm{~m}, 3 \mathrm{H}), 1.24(\mathrm{~d}, J=3.7 \mathrm{~Hz}$, $3 \mathrm{H}), 1.09(\mathrm{~s}, 6 \mathrm{H}) ;{ }^{13} \mathrm{C}$ NMR $\left(125 \mathrm{MHz}, \mathrm{DMSO}-d_{6}\right) \delta$ :
$179.02(\mathrm{C}=\mathrm{S}), 170.61(\mathrm{C}=\mathrm{O}), 149.82(\mathrm{C}=\mathrm{N}), 148.82$, $146.61,129.96,128.45,123.00,122.85,60.37,49.60$, $42.35,37.70,37.43,36.79,36.38,33.69,24.44,24.11$, 23.74, 23.44, 23.28, 18.69, 18.08, 14.17; IR (KBr) v: 3457, 3299, 2958, 2926, 2866, 1718, 1651, 1490, 1283, 1103, 1081, $556 \mathrm{~cm}^{-1}$; ESI-MS $(+) \mathrm{m} / \mathrm{z}: 415.2[\mathrm{M}+\mathrm{H}]^{+}$; HRMS (EI) calcd for $\mathrm{C}_{23} \mathrm{H}_{34} \mathrm{~N}_{4} \mathrm{OS}$ 415.2536, found 415.2532 .

乙酰脱氢枞胺-7-异烟酰腙 $(\mathbf{3 e})$ : 淡黄色固体，产率 65\%. m.p. $157.3 \sim 158.6{ }^{\circ} \mathrm{C} ;{ }^{1} \mathrm{H}$ NMR (500 MHz, $\mathrm{CDCl}_{3}$ ) $\delta: 10.07$ (s, 1H, NNHC=O), 8.75 (s, 2H, Py-H), 7.89 (d, $J=26.3 \mathrm{~Hz}, 3 \mathrm{H}, \mathrm{Py}-\mathrm{H}, \mathrm{ArH}), 7.21$ (d, $J=23.3 \mathrm{~Hz}, \mathrm{ArH})$, $5.80(\mathrm{~s}, 1 \mathrm{H}, \mathrm{NH}), 2.99 \sim 3.75(\mathrm{~m}, 3 \mathrm{H}), 2.33(\mathrm{~d}, J=10.7$ $\mathrm{Hz}, 2 \mathrm{H}), 2.04$ (s, 2H), $1.91(\mathrm{~s}, 3 \mathrm{H}), 1.75 \sim 1.67$ (m, 2H), $1.47 \sim 1.28(\mathrm{~m}, 3 \mathrm{H}), 1.26 \sim 1.17(\mathrm{~m}, 9 \mathrm{H}), 1.03(\mathrm{~d}, J=19.4$ $\mathrm{Hz}, 3 \mathrm{H}) ;{ }^{13} \mathrm{C} \mathrm{NMR}\left(125 \mathrm{MHz}, \mathrm{CDCl}_{3}\right) \delta: 170.95\left(\mathrm{COCH}_{3}\right)$, 157.47 (NHCO), $150.32(\mathrm{C}=\mathrm{N}), 149.46$ (Py-C), 149.32 (Py-C), 146.58 (C-9), 141.43 (C-13), 141.23 (Рy-C), 128.74 (C-12), 124.00 (C-8), 123.82 (C-14), 123.60 (C-11), 122.40 (Рy-C), 121.64 (Рy-C), 49.35, 42.09, 37.72, $37.63,37.36,36.57,33.67,23.94,23.77,23.49,23.28$, 18.91, 18.40, 18.14; IR (KBr) v: 3424, 2959, 2921, 2868, 1736, 1656, 1527, 1382, 1283, $1140 \mathrm{~cm}^{-1}$; ESI-MS (+) $m / z: 461.4[\mathrm{M}+\mathrm{H}]^{+}$; HRMS (EI) calcd for $\mathrm{C}_{28} \mathrm{H}_{36} \mathrm{~N}_{4} \mathrm{O}_{2}$ 461.2921, found 461.2917.

三氟乙酰脱氢枞胺-7-肜(3f)：淡黄色固体，产率 65\%, m.p. 160.8 161.9 ${ }^{\circ} \mathrm{C} ;{ }^{1} \mathrm{H}$ NMR (500 MHz, $\mathrm{CDCl}_{3}$ ) $\delta: 7.70(\mathrm{~s}, 1 \mathrm{H}, \operatorname{ArH}), 7.24 \sim 7.17(\mathrm{~m}, 2 \mathrm{H}, \operatorname{ArH}), 6.47(\mathrm{~s}$, $1 \mathrm{H}, \mathrm{NH}), 3.46(\mathrm{dd}, J=13.7,7.5 \mathrm{~Hz}, 1 \mathrm{H}), 2.99 \sim 3.13(\mathrm{~m}$, $2 \mathrm{H}), 2.68 \sim 2.90(\mathrm{~m}, 2 \mathrm{H}), 2.28(\mathrm{t}, J=11.6 \mathrm{~Hz}, 1 \mathrm{H}), 1.68 \sim$ $1.73(\mathrm{~m}, 2 \mathrm{H}), 1.57(\mathrm{~d}, J=4.8 \mathrm{~Hz}, 1 \mathrm{H}), 1.42 \sim 1.54(\mathrm{~m}$, $3 \mathrm{H}), 1.26 \sim 1.38(\mathrm{~m}, 7 \mathrm{H}), 1.12(\mathrm{~s}, 3 \mathrm{H}), 1.11 \sim 1.07(\mathrm{~m}$, $3 \mathrm{H}) ;{ }^{13} \mathrm{C}$ NMR $\left(125 \mathrm{MHz}, \mathrm{CDCl}_{3}\right) \delta: 157.59\left(\mathrm{COCF}_{3}\right)$, $155.43(\mathrm{C}=\mathrm{N}), 148.63(\mathrm{C}-9), 146.71 \quad(\mathrm{C}-13), 128.53$ (C-12), 128.17 (C-8), 123.00 (C-14), 122.15 (C-11), $114.77\left(\mathrm{CF}_{3}\right)$, 50.18, 42.82, 37.78, 37.39, 37.04, 36.12, 33.66, 23.99, 23.77, 23.11, 22.02, 18.15, 18.01; IR (KBr) $v: 3330,3119,2961,2931,2871,1707,1561,1386,1209$, 1161, 946, $722 \mathrm{~cm}^{-1}$; ESI- MS (+) $m / z: 411.2[\mathrm{M}+\mathrm{H}]^{+}$, $433.2[\mathrm{M}+\mathrm{Na}]^{+}$; HRMS (EI) calcd for $\mathrm{C}_{22} \mathrm{H}_{29} \mathrm{~F}_{3} \mathrm{~N}_{2} \mathrm{O}_{2}$ 411.2261, found 411.2259.

三氟乙酰脱氢枞胺-7-氨基脲酰腙(3g): 白色粉末, 产率 78\%0 m.p. 143.2 144.5 ${ }^{\circ} \mathrm{C} ;{ }^{1} \mathrm{H}$ NMR $(500 \mathrm{MHz}$, $\left.\mathrm{CDCl}_{3}\right) \delta: 10.34(\mathrm{~s}, 1 \mathrm{H}, \mathrm{NNHC}=\mathrm{S}), 7.76(\mathrm{~s}, 1 \mathrm{H}, \mathrm{ArH})$, 7.19 (s, 2H, ArH), 6.77 (s, 1H, NH), 6.25 5.69 (m, 2H, 
$\left.\mathrm{NH}_{2}\right), 3.00 \sim 2.81(\mathrm{~m}, 3 \mathrm{H}), 2.58(\mathrm{dd}, J=18.5,13.4 \mathrm{~Hz}$, $1 \mathrm{H}), 2.30(\mathrm{~d}, J=12.6 \mathrm{~Hz}, 1 \mathrm{H}), 1.83 \sim 1.75(\mathrm{~m}, 2 \mathrm{H}), 1.67 \sim$ $1.57(\mathrm{~m}, 3 \mathrm{H}), 1.29 \sim 1.22(\mathrm{~m}, 8 \mathrm{H}), 1.19 \sim 1.11(\mathrm{~m}, 6 \mathrm{H})$; ${ }^{13} \mathrm{C}$ NMR $\left(125 \mathrm{MHz}, \mathrm{CDCl}_{3}\right) \delta: 159.20\left(\mathrm{COCF}_{3}\right), 157.64$ $\left(\mathrm{CONH}_{2}\right), 148.51$ (C-9), 146.64 (C-13), 145.88 (C-12), 130.74 (C-8), 127.55 (C-14), 122.77 (C-11), $122.49\left(\mathrm{CF}_{3}\right)$, $51.35,44.77,38.14,37.40,36.81,36.27,33.75,24.69$, 24.09, 23.80, 23.01, 18.08, 17.07; IR (KBr) v: 3500, 3450, 3377, 1961, 2929, 2870, 1724, 1689, 1670, 1569, 1421, $1121,1155 \mathrm{~cm}^{-1}$; ESI-MS (+) $m / z: 453.3[\mathrm{M}+\mathrm{H}]^{+}, 475.4$ $[\mathrm{M}+\mathrm{Na}]^{+}$; HRMS (EI) calcd for $\mathrm{C}_{23} \mathrm{H}_{31} \mathrm{~F}_{3} \mathrm{~N}_{4} \mathrm{O}_{2} 453.2481$, found 453.2477 .

三氟乙酰脱氢枞胺-7-异烟酰腙(3h): 淡黄色粉末, 产率 74\%. m.p. $182.2 \sim 183.5{ }^{\circ} \mathrm{C}$; ${ }^{1} \mathrm{H}$ NMR $(500 \mathrm{MHz}$, $\left.\mathrm{CDCl}_{3}\right) \delta: 10.17(\mathrm{~s}, 1 \mathrm{H}, \mathrm{NNHC}=\mathrm{O}), 8.73(\mathrm{~d}, J=5.2 \mathrm{~Hz}$, $2 \mathrm{H}, \mathrm{PyH}), 7.77$ (d, $J=5.6 \mathrm{~Hz}, 2 \mathrm{H}, \mathrm{PyH}), 7.60(\mathrm{~s}, 1 \mathrm{H}$, $\operatorname{ArH}), 7.15$ (d, $J=17.4 \mathrm{~Hz}, 2 \mathrm{H}, \mathrm{ArH}), 6.62(\mathrm{~s}, 1 \mathrm{H}, \mathrm{NH})$, $4.42 \sim 3.30(\mathrm{~m}, 2 \mathrm{H}), 3.12 \sim 2.94(\mathrm{~m}, 1 \mathrm{H}), 2.92 \sim 2.76(\mathrm{~m}$, $2 \mathrm{H}), 1.85 \sim 1.76(\mathrm{~m}, 2 \mathrm{H}), 1.54 \sim 1.68(\mathrm{~m}, 2 \mathrm{H}), 1.50(\mathrm{~s}$, $1 \mathrm{H}), 1.31(\mathrm{~d}, \quad J=18.0 \mathrm{~Hz}, 1 \mathrm{H}), 1.25(\mathrm{~s}, 1 \mathrm{H}), 1.20(\mathrm{t}, J=$ $7.8 \mathrm{~Hz}, 6 \mathrm{H}), 1.13$ (s, 3H), 1.07 (s, 3H); ${ }^{13} \mathrm{C}$ NMR $(125$ $\left.\mathrm{MHz}, \mathrm{CDCl}_{3}\right) \delta: 169.24$ (NHCO), $157.04\left(\mathrm{COCF}_{3}\right), 150.19$ (Py-C), 149.54 (Py-C), 149.22 (C-9), 148.99 (C-13), $146.63(\mathrm{C}=\mathrm{N}), 141.34($ Py-C), $128.90(\mathrm{C}-12), 123.84$ (C-8), 123.23 (C-14), 122.88 (C-11), 122.37 (Рy-C), 121.42 (Py-C), $113.90\left(\mathrm{CF}_{3}\right), 60.31,49.80,42.79,37.89$, 37.17, 36.11, 33.39, 23.69, 23.57, 23.41, 18.31, 17.94, 14.09; IR (KBr) v: 3424, 3255, 1961, 2927, 2870, 1720, 1660, 1385, 1208, $1152 \mathrm{~cm}^{-1}$; ESI-MS (+) m/z: 515.3 $[\mathrm{M}+\mathrm{H}]^{+}, 537.3[\mathrm{M}+\mathrm{Na}]^{+}$; HRMS (EI) calcd for $\mathrm{C}_{28} \mathrm{H}_{33} \mathrm{~F}_{3} \mathrm{~N}_{4} \mathrm{O}_{2}$ 515.2638, found 515.2634.

\subsection{3 目标化合物 $\mathbf{4 a}$ 和 $\mathbf{4 b}$ 的合成}

以目标化合物 $4 \mathbf{a}$ 的合成为例, 将 $3 \mathrm{mmol}$ 的 $\mathbf{2 a}$ 用 $40 \mathrm{~mL}$ 醋酸溶解加入 $250 \mathrm{~mL}$ 四口烧瓶, 升温到 $70{ }^{\circ} \mathrm{C}$, 然后将 $10 \mathrm{mmol}$ 的二氧化硒用醋酸溶解, 缓慢滴加到四 口烧瓶中, 滴加完毕, 升温到回流, 继续反应 $5 \mathrm{~h}$. 反应 结束后过滤除去反应生成的硒粉, 滤液减压蒸除溶剂, 残留物后用硅胶柱层析[洗脱剂: $V$ (石油醚) : $V$ ( 乙酸乙 酯) $=7: 3$ ]分离得到目标产物. 用类似的方法得到产物 $4 \mathrm{~b}$.

乙酰脱氢枞胺-(5,6-烯)-7-唒(4a): 淡黄色固体, 产 率 50\%. m.p. $123.1 \sim 124.3{ }^{\circ} \mathrm{C} ;{ }^{1} \mathrm{H}$ NMR $(500 \mathrm{MHz}$, $\left.\mathrm{CDCl}_{3}\right) \delta: 7.91(\mathrm{~d}, J=7.2 \mathrm{~Hz}, 1 \mathrm{H}, \mathrm{ArH}), 7.44(\mathrm{~s}, 2 \mathrm{H}$, ArH), 6.40 (s, 1H, H-6), 5.80 (s, 1H, CONH), 3.68 (dd, $J=14.3,9.0 \mathrm{~Hz}, 1 \mathrm{H}), 3.34(\mathrm{dd}, J=14.2,3.0 \mathrm{~Hz}, 1 \mathrm{H})$,
$3.01 \sim 2.86(\mathrm{~m}, 2 \mathrm{H}), 2.11 \sim 1.98(\mathrm{~s}, 1 \mathrm{H}), 1.94(\mathrm{~d}, J=12.6$ $\mathrm{Hz}, 3 \mathrm{H}, 3 \mathrm{H}), 1.89 \sim 1.70(\mathrm{~m}, 1 \mathrm{H}), 1.70 \sim 1.58(\mathrm{~m}, 3 \mathrm{H})$, $1.52(\mathrm{~s}, 3 \mathrm{H}), 1.37(\mathrm{~s}, 3 \mathrm{H}), 1.30 \sim 1.23(\mathrm{~m}, 6 \mathrm{H}) ;{ }^{13} \mathrm{C} \mathrm{NMR}$ (125 MHz, $\left.\mathrm{CDCl}_{3}\right) \delta: 185.25$ (C-7), 170.99 (NHCO), 170.38 (C-5), 150.97 (C-9), 147.13 (C-13), 131.52 (C-12), 129.92 (C-14), 124.80 (C-11), 124.55 (C-6), 123.53 (C-8), 48.54, 41.87, 41.19, 35.96, 34.08, 34.03, 33.58, 27.01, 23.75, 23.69, 23.30, 17.62; IR (KBr) $v: 3360,3072,2960$, 2931, 2869, 1652, 1607, 1545, 1495, 1276, 1099, 885, 596 $\mathrm{cm}^{-1}$; ESI-MS (+) $m / z: 340.1[\mathrm{M}+\mathrm{H}]^{+}, 360.1[\mathrm{M}+\mathrm{Na}]^{+}$; HRMS (EI) calcd for $\mathrm{C}_{22} \mathrm{H}_{29} \mathrm{NO}_{2}$ 340.2277, found 340.2280

三氟乙酰脱氢枞胺-(5,6-烯)-7-酮(4b): 淡黄色固体, 产率 45\%. m.p. 95.1 96.3 ${ }^{\circ} \mathrm{C} ;{ }^{1} \mathrm{H}$ NMR $(500 \mathrm{MHz}$, $\left.\mathrm{CDCl}_{3}\right) \delta: 7.96(\mathrm{t}, J=6.1 \mathrm{~Hz}, 1 \mathrm{H}, \mathrm{ArH}), 7.49 \sim 7.41(\mathrm{~m}$, $2 \mathrm{H}, \mathrm{ArH}), 6.38(\mathrm{~s}, 2 \mathrm{H}, \mathrm{H}-6, \mathrm{NH}), 3.76 \sim 3.63(\mathrm{~m}, 1 \mathrm{H}), 3.51$ (dd, $J=14.2,3.8 \mathrm{~Hz}, 1 \mathrm{H}), 2.97$ (dd, $J=14.5,7.0 \mathrm{~Hz}, 1 \mathrm{H})$, $2.68 \sim 2.52(\mathrm{~m}, 1 \mathrm{H}), 2.13 \sim 2.00(\mathrm{~m}, 1 \mathrm{H}), 1.94 \sim 1.77(\mathrm{~m}$, $1 \mathrm{H}), 1.72 \sim 1.58(\mathrm{~m}, 3 \mathrm{H}), 1.57 \sim 1.50(\mathrm{~m}, 4 \mathrm{H}), 1.44 \sim 1.40$ (m, 3H), $1.31 \sim 1.24(\mathrm{~m}, 7 \mathrm{H}) ;{ }^{13} \mathrm{C}$ NMR $(125 \mathrm{MHz}$, $\left.\mathrm{CDCl}_{3}\right) \delta: 185.02$ (C-7), 169.59 (C-5), 157.83 (NHCO), 150.71 (C-9), 147.39 (C-13), 131.79 (C-12), 129.79 (C-8), 124.73 (C-11), 124.59 (C-14), 123.78 (C-6), 48.79, 41.67, $41.20,35.86,34.06,34.00,33.66,26.78,23.75,17.52$; IR (KBr) $v: 3326,2960,2934,2817,1773,1650,1559,1458$, 1358, 1209, 1161, $757 \mathrm{~cm}^{-1}$; ESI-MS (+) $\mathrm{m} / \mathrm{z}: 394.4$ $[\mathrm{M}+\mathrm{H}]^{+}, 416.4[\mathrm{M}+\mathrm{Na}]^{+}$; HRMS (EI) calcd for $\mathrm{C}_{22} \mathrm{H}_{26} \mathrm{~F}_{3} \mathrm{NO}_{2}$ 394.1994, found 394.1997.

\subsection{4 目标化合物 $\mathbf{5}$ 的合成}

参照文献[21]先合成三乙酰基没食子酰氯. 取 17.5 $\mathrm{mmol}$ 脱氢枞胺(1)用 $60 \mathrm{~mL}$ 二氯甲烷溶解后加入 $250 \mathrm{~mL}$ 四口烧瓶中, 再取 $18 \mathrm{mmol}$ 的三乙酰基没食子酰氯用 20 $\mathrm{mL}$ 二氯甲烷溶解, 并缓慢滴加到四口烧瓶中, 滴加完 毕, 室温下搅拌反应 $4 \mathrm{~h}$. 反应结束后减压蒸除溶剂, 残 留物用硅胶柱层析 [洗脱剂: $V$ (石油醚) $: V($ 乙酸乙酯 $)=$ $6: 4]$ 分离得到目标产物 $N$ - $(3,4,5$-三乙酰氧基苯甲酰 基)-脱氢枞胺(5). 白色固体, 产率 89\%. m.p. 195.1 $196.3{ }^{\circ} \mathrm{C} ;{ }^{1} \mathrm{H}$ NMR $\left(500 \mathrm{MHz}, \mathrm{CDCl}_{3}\right) \delta: 7.49 \sim 7.46(\mathrm{~m}$, $2 \mathrm{H}, \mathrm{ArH}), 7.16$ (d, $J=8.2 \mathrm{~Hz}, 1 \mathrm{H}, \mathrm{ArH}), 6.98$ (dd, $J=8.1$, $1.7 \mathrm{~Hz}, 1 \mathrm{H}, \mathrm{ArH}), 6.88(\mathrm{~s}, 1 \mathrm{H}, \mathrm{ArH}), 6.12(\mathrm{~d}, J=20.8 \mathrm{~Hz}$, $1 \mathrm{H}, \mathrm{CONH}), 3.32(\mathrm{t}, J=7.9 \mathrm{~Hz}, 2 \mathrm{H}), 3.03 \sim 2.91(\mathrm{~m}, 1 \mathrm{H})$, $2.82 \sim 2.77(\mathrm{~m}, 2 \mathrm{H}), 2.27(\mathrm{~s}, 9 \mathrm{H}, \mathrm{OAc}), 1.94 \sim 1.78(\mathrm{~m}$, $1 \mathrm{H}), 1.71 \sim 1.67(\mathrm{~m}, 1 \mathrm{H}), 1.48 \sim 1.64(\mathrm{~m}, 2 \mathrm{H}), 1.45 \sim 1.38$ $(\mathrm{m}, 2 \mathrm{H}), 1.37 \sim 1.34(\mathrm{~m}, 1 \mathrm{H}), 1.31 \sim 1.26(\mathrm{~m}, 1 \mathrm{H}), 1.24(\mathrm{~d}$, $J=7.2 \mathrm{~Hz}, 1 \mathrm{H}), 1.22$ (s, 6H), 1.21 (s, 3H), 0.98 (s, 3H); 
${ }^{13} \mathrm{C}$ NMR (125 MHz, $\left.\mathrm{CDCl}_{3}\right) \delta: 167.56\left(\mathrm{CH}_{3} \mathrm{CO}\right), 167.54$ $\left(\mathrm{CH}_{3} \mathrm{CO}\right), 166.42\left(\mathrm{CH}_{3} \mathrm{CO}\right), 165.30(\mathrm{NHCO}), 146.96$, 145.57 (C-9), 143.48, 137.10, 134.67 (C-13), 133.12, 126.93 (C-8), 125.95 (C-14), 124.10 (C-11), 123.80 (C-12), 119.45, 114.94, 50.71, 46.03, 38.28, 37.63, 37.51, $36.35,33.38,30.26,25.36,23.92,23.89,20.51,20.07$, 19.85, 19.51, 19.08, 18.54; IR (KBr) v: 3442, 2931, 2869, 1776, 1658, 1489, 1375, 1222, 1188, 1053, $550 \mathrm{~cm}^{-1}$; ESI-MS (+) $m / z: 564.2[\mathrm{M}+\mathrm{H}]^{+}, 586.2[\mathrm{M}+\mathrm{Na}]^{+}$; HRMS (EI) calcd for $\mathrm{C}_{33} \mathrm{H}_{41} \mathrm{NO}_{7}$ 564.2966, found 564.2975.

\subsection{5 目标化合物 $\mathbf{6}$ 的合成}

取 $10 \mathrm{mmol}$ 化合物 5 用 $60 \mathrm{~mL}$ 二氯甲烷溶解, 加入 $250 \mathrm{~mL}$ 四口烧瓶, 然后往四口烧瓶中滴加两滴水合肼, 在室温下摚拌反应 $30 \mathrm{~min}$, 反应结束后用 $30 \mathrm{~mL}$ 水洗 3 次, 将下层液减压蒸馏得到产物 $N$ - $(3,4,5$-三差基苯甲酰 基)-脱氢枞胺(6). 淡黄色固体, 产率 70\%. m.p. 153.5 $154.7{ }^{\circ} \mathrm{C} ;{ }^{1} \mathrm{H}$ NMR $\left(500 \mathrm{MHz}, \mathrm{CDCl}_{3}\right) \delta: 7.17 \sim 7.03(\mathrm{~m}$, $1 \mathrm{H}, \mathrm{ArH}), 6.92$ (t, $J=15.0 \mathrm{~Hz}, 1 \mathrm{H}, \mathrm{ArH}), 6.81$ (t, $J=15.7$ $\mathrm{Hz}, 3 \mathrm{H}, \mathrm{ArH}), 6.39(\mathrm{~s}, 1 \mathrm{H}, \mathrm{NH}), 5.67 \sim 5.43(\mathrm{~m}, 3 \mathrm{H}, \mathrm{OH})$, $3.70(\mathrm{q}, J=7.0 \mathrm{~Hz}, 1 \mathrm{H}), 3.40(\mathrm{~s}, 1 \mathrm{H}), 3.06(\mathrm{~s}, 1 \mathrm{H}), 2.84 \sim$ $2.77(\mathrm{~m}, 2 \mathrm{H}), 1.83 \sim 1.78(\mathrm{~m}, 1 \mathrm{H}), 1.66(\mathrm{~s}, 2 \mathrm{H}), 1.57(\mathrm{~s}$, $1 \mathrm{H}), 1.34 \sim 1.44(\mathrm{~m}, 2 \mathrm{H}), 1.31 \sim 1.26(\mathrm{~m}, 1 \mathrm{H}), 1.22 \sim 1.24$ $(\mathrm{m}, 2 \mathrm{H}), 1.20 \sim 1.18(\mathrm{~m}, 6 \mathrm{H}), 1.13(\mathrm{~s}, 3 \mathrm{H}), 0.92 \sim 0.85(\mathrm{~m}$, $3 \mathrm{H}) ;{ }^{13} \mathrm{C}$ NMR (125 MHz, $\left.\mathrm{CDCl}_{3}\right) \delta: 169.33$ (NHCO), 146.91, 145.64 (C-9), 145.55 (C-13), 144.82, 136.20, 134.53 (C-8), 126.87, 125.23 (C-14), 124.11 (C-11), 123.80 (C-12), 107.46, 107.25, 51.20, 46.71, 38.30, 37.67, $37.47,36.35,33.39,30.22,25.26,23.94,23.80,19.12$, 18.54, 18.05; IR (KBr) v: 3334, 2957, 2926, 2867, 1603, 1519, 1501, 1443, 1374, 1342, 1281, 1201, $1038 \mathrm{~cm}^{-1}$; ESI-MS (+) $m / z: 438.2[\mathrm{M}+\mathrm{H}]^{+}, 460.2[\mathrm{M}+\mathrm{Na}]^{+}$; HRMS (EI) calcd for $\mathrm{C}_{27} \mathrm{H}_{35} \mathrm{NO}_{4}$ 438.2647, found 438.2644 .

\section{3 抗自由基活性测试}

\subsection{1 清除超氧阴离子 $\left(\mathrm{O}_{2}^{-}\right)$实验}

参考文献[22，23]方法测试目标化合物清除超氧阴 离子 $\left(\mathrm{O}_{2}^{-}\right)$的能力. 在 $10 \mathrm{~mL}$ 的比色典中加入 $5.7 \mathrm{~mL}$ 浓 度 $50 \mathrm{mmol} / \mathrm{L}$ 的 Tris- $\mathrm{HCl}$ 缓冲液 $(\mathrm{pH} 8.20$ ), 再加入 0.2 $\mathrm{mL}$ 浓度为 $0.1000 \mathrm{~g} / \mathrm{L}$ 的样品, 于 $25{ }^{\circ} \mathrm{C}$ 保温 $10 \mathrm{~min}$, 然 后加入 $25{ }^{\circ} \mathrm{C}$ 预热的 $6 \mathrm{mmol} / \mathrm{L}$ 邻苯三酚 $0.1 \mathrm{~mL}$, 总体积 $6.0 \mathrm{~mL}$, 迅速摇匀后用紫外可见分光光度计测定反应 1 $\min$ 时 $320 \mathrm{~nm}$ 处的吸光度, 此后每隔 $30 \mathrm{~s}$ 读取一次吸光 度 $A$, 到 $300 \mathrm{~s}$ 时停止. 以 Tris- $\mathrm{HCl}$ 缓冲液为空白调零, 无样品液为阳性对照, 测试所有样品. 样品对 $\mathrm{O}_{2}^{-}$的抑
制率按如下公式计算:

$$
\text { 抑制率 }=\left[(\Delta A / \Delta t)_{\text {对照 }}-(\Delta A / \Delta t)\right] /(\Delta A / \Delta t)_{\text {对照 }} \times 100 \%
$$

\subsection{2 清除二苯代苦味酰基自由基(DPPH•)实验}

参考文献[24 27]方法测试目标化合物清除二苯代 苦味酰基自由基(DPPH•)的能力. 分别配制不同浓度的 样品溶液, 取 $2 \mathrm{~mL}$ 的样品溶液与比色管中, 再加入 $8.62 \times 10^{-5} \mathrm{~mol} / \mathrm{L}$ 的 DPPH 溶液 $2 \mathrm{~mL}$, 使混合液总体积 为 $4 \mathrm{~mL}$, 充分摇匀在室温下反应 $30 \mathrm{~min}$ 后用紫外分光 光度计测定 $517 \mathrm{~nm}$ 处的吸光度 $A$ (用样品溶液调零后测 量). 在 $2 \mathrm{~mL} \mathrm{DPPH}$ 溶液中加入 $2 \mathrm{~mL}$ 乙醇溶液, 测定吸 光度 $A_{0}$. 样品对 $\mathrm{DPPH} \bullet$ 的清除率计算公式如下:

清除率 $=\left[\left(A_{0}-A\right) / A_{0}\right] \times 100 \%$

\section{2 结果与讨论}

\section{1 合成方法}

实验先通过在脱氢枞胺 7 位引入羰基, 成功合成了 一系列含 Schiff 和酰腙基团的新型脱氢枞胺衍生物, 研 究过程中发现加入少量醋酸作催化剂能有效地促进反 应的进行，以化合物 3e 的合成为例，在未加催化剂时， 反应 $12 \mathrm{~h}$ 收率只有 $30 \%$, 但加入醋酸后反应 $5 \mathrm{~h}$ 收率高 达 $85 \%$.

在对 $4 a \sim 4 b$ 的合成研究中, 预期结果是在脱氢枞 胺 6 位再引入羰基, 实际产物却是在 5,6 位形成了烯键, 尝试多种方法未能在 6 位引入羰基, 烯键的形成说明氧 化产生的中间产物不稳定，中间产物经脱氢形成烯键. 另外在产物 6 的合成研究中, 原先设计路线是没食子酸 直接和脱氢枞胺反应，实际操作中发现没食子酸苯环上 的三个羟基在反应过程中容易变异，因此采用了现在的 合成方法.

\section{2 抗自由基测试结果与分析}

对合成化合物的抗自由基活性测试结果如表 1. 实 验结果发现，脱氢枞胺本身具有一定清除自由基能力, 但不是很强, 其对 $(\mathrm{DPPH} \cdot)$ 的清除能力 $\left(\mathrm{IC}_{50}=4.580 \times\right.$ $\left.10^{3} \mathrm{mg} / \mathrm{L}\right)$ 比没食子酸 $\left(\mathrm{IC}_{50}=0.001 \times 10^{3} \mathrm{mg} / \mathrm{L}\right)$ 弱很多. 从化合物 5 和 $\mathbf{6}$ 的测试结果可以看出引入多羟基的没食 子酸能够大幅度提高脱氢枞胺抗自由基能力, 特别是化 合物 6, 其对超氧阴离子 $\left(\mathrm{O}_{2}^{-}\right)$的抑制率为 $38.18 \%$, 几乎 是 $\mathrm{Vc}(23.25 \%)$ 的两倍, 对自由基( $\mathrm{DPPH} \bullet$ ) 的清除能力 $\left(\mathrm{IC}_{50}=0.002 \times 10^{3} \mathrm{mg} / \mathrm{L}\right)$ 比 $\mathrm{Vc}\left(\mathrm{IC}_{50}=0.236 \times 10^{3} \mathrm{mg} / \mathrm{L}\right)$ 强 118 倍, 化合物 5 因为羟基被羧基保护使得清除自由 基能力有所下降; 化合物 3c 和 3d 对 (DPPH•)的半数清 除浓度分别为 $3.124 \times 10^{3}$ 和 $0.087 \times 10^{3} \mathrm{mg} / \mathrm{L}$ ，对比后可 以发现 $\mathrm{C}=\mathrm{S}$ 键清除 (DPPH•)自由基能力很强; 化合物 
3f 和 $3 \mathbf{h}$ 对(DPPH•)的半数清除浓度分别为 $0.325 \times 10^{3}$, $0.223 \times 10^{3} \mathrm{mg} / \mathrm{L}$, 表明肜基和异烟基对(DPPH•)自由基 有较好的清除作用; 化合物 $\mathbf{3 c}$ 和 $\mathbf{3 g}$ 对 $\mathrm{O}_{2}^{-}$抑制率分别 为 $0.86 \%$ 和 $19.25 \%$, 对比中可以发现 $\mathrm{CF}_{3}$ 对 $\mathrm{O}_{2}^{-}$有一定 的抑制作用, 3e (5.35\%)和 3h (10.91\%)的对比也说明了 这点.

综上所述, 本实验的研究确认了脱氢枞胺清除自由 基的能力, 并且合成的几种新型脱氢枞胺衍生物表现出 良好的抗自由基性能, 为后续研究提供了方向和依据, 为拓展脱氢枞胺的应用开辟了新的途径.

表 1 化合物清除自由基活性 ${ }^{a}$

Table 1 Scavenging free radicals activity of the target compounds

\begin{tabular}{lcc}
\hline Compd. & $\mathrm{O}_{2}^{-}$抑制率 $/ \%$ & 清除 $\mathrm{DPPH} \cdot$ 能力 $\mathrm{IC}_{50} /\left(\mathrm{mg}^{\circ} \mathrm{L}^{-1}\right)$ \\
\hline 脱氢枞胺 & 23.21 & $4.580 \times 10^{3}$ \\
$\mathbf{2 a}$ & 7.87 & $0.598 \times 10^{3}$ \\
$\mathbf{3 a}$ & 2.46 & $>5.000 \times 10^{3}$ \\
$\mathbf{3 b}$ & 11.87 & $>5.000 \times 10^{3}$ \\
$\mathbf{3 c}$ & 0.86 & $3.124 \times 10^{3}$ \\
$\mathbf{3 d}$ & 24.71 & $0.087 \times 10^{3}$ \\
$\mathbf{3 e}$ & 5.35 & $0.555 \times 10^{3}$ \\
$\mathbf{3 f}$ & 14.01 & $0.325 \times 10^{3}$ \\
$\mathbf{3 g}$ & 19.25 & $2.988 \times 10^{3}$ \\
$\mathbf{3 h}$ & 10.91 & $0.223 \times 10^{3}$ \\
$\mathbf{4 a}$ & 7.56 & $0.586 \times 10^{3}$ \\
$\mathbf{4 b}$ & 8.54 & $0.577 \times 10^{3}$ \\
$\mathbf{5}$ & 28.98 & $0.067 \times 10^{3}$ \\
$\mathbf{6}$ & 38.18 & $0.002 \times 10^{3}$ \\
没食子酸 & 10.11 & $0.001 \times 10^{3}$ \\
$\mathrm{Vc}$ & 18.35 & $0.236 \times 10^{3}$ \\
\hline
\end{tabular}

${ }^{a}$ 表中 $\mathrm{Vc}$ 的测得值与文献[28]报道相近.

\section{References}

[1] Stuart, O.; Philip, B. Account. Org. Soc. 1985, 10, 211.

[2] Cumming, R. B.; Marva, F. W. Food Cosmet. Toxicol. 1973, 11, 547.

[3] Wang, Y.; Yang, C.-G.; Zhou, Y.-H. Nat. Prod. Res. Dev. 1997, 4, 59 (in Chinese). (王延, 杨成根, 周永红, 天然产物研究与开发, 1997, 4, 59.)

[4] Zhou, Z.; Lin, Z. X. Molecules 2012, 17, 4209.

[5] Lei, Q.; Feng, S.-B.; Zhang, Y. Chin. J. Org. Chem. 2011, 31, 1468 (in Chinese).
(雷茜, 冯少波, 张业, 有机化学, 2011, 31, 1468.)

[6] Sui, Y.; Liu, D. S.; Hu, R. H. J. Mater. Chem. 2011, 21, 38.

[7] Wendell, W.; Wilkerson, W. G.; Indawati, D.; Ricard, R. H. Bioorg. Med. Chem. Lett. 1993, 3, 2087.

[8] Cristiano, B.; Laura, F.; Barbara, M.; Marco P.; Ermanno, V. Tetrahedron: Asymmetry 2003, 14, 3779.

[9] Bang, S. D.; Johnson, S. K.; Park, J. C. S. US 5248696, 1993 [Chem. Abstr. 1993, 118, 32926].

[10] Lin, L. Y.; Bao, Y. L.; Chen, Y.; Sun, L. G.; Yang, X. G.; Liu, B.; Lin, Z. X.; Zhang, Y. W.; Yu, C. L.; Wu, Y.; Li, Y. X. Chem. Biol. Interact. 2012, 199, 63.

[11] Stefan, M.; Gudrun, M. Eur. J. Biochem. 1974, 47, 469.

[12] Tuanjai, N.; Supalax, S.; Thawatchai, T.; Wittaya, N. Food Res. Int. 2011, 44, 798.

[13] Pantelidis, G. E.; Vasilakakis, M.; Manganaris, G. A.; Diamantidis, G. Food Chem. 2007, 102, 777.

[14] Wang, Q.-R.; Zhang, R.-M.; He, X.-Y.; Zhen, J.-H. J. Shantou Univ. Med. College 2010, 23, 193 (in Chinese).

(王钦荣, 张鲁勉, 何小英, 郑锦鸿, 汕头大学医学院学报, 2010 , 23, 193.)

[15] Okezie, I.; Aruoma, A.; Murcia, J.; Butler, B.; Halliwell, B. J. Agric. Food Chem. 1993, 41, 1880.

[16] Türkkan, B.; Özyürek, M.; Bener, M.; Güçlü, K.; Reşat, A. Chem. Biol. Int. 2009, 177, 153

[17] Thomas, P. A.; Alan, W.; Terry I.; Frank, A. M.; Charles, C.; Donald, V. G.; David, W. Ind. Crops Prod. 2002, 16, 43

[18] Girish, B.; Maru, S. V.; Bhide, V. Cancer Lett. 1982, 17, 75.

[19] Chen, Y.; Guo, B.; Zhang, D.-H.; Zhou, J. Chem. Ind. Times 2009, 23, 71 (in Chinese). (陈泳，郭斌，张丹红，周洁，化工时刊， 2009, 23, 71.)

[20] Chen, Y.; Lin, Z.-X. J. Nanjing Forestry Univ. (Nat. Sci. Ed.) 2012, 36, 101 (in Chinese).

(陈泳, 林中祥, 南京林业大学学报(自然科学版), 2012, 36, 101.)

[21] Zhang, W.-J.; Xiong, D.-Y.; Liu, X.-M. Appl. Chem. Ind. 2010, 39, 1894 (in Chinese). (张文君, 熊德元, 刘雄民, 应用化工, 2010, 39, 1894.)

[22] El-Toumy, S. A.; El-Souda, S. S.; Mohamed, T. K.; Brouard, I.; Bermejo, J. Carbohydr. Res. 2012, 360, 47.

[23] Chamundeeswari, D.; Vasantha, G.; Gopalakrishnan, S.; Sukumar, E. J. Ethnopharmacol. 2003, 88, 51.

[24] Andrzej, L.; Dawidowicz, D. W.; Małgorzata, O. Food Chem. 2012, 131, 1037.

[25] Shizuka, S.; Jun, K. Tetrahedron 2005, 61, 8101.

[26] Oyais, A. C.; Muzaffar, H. N.; Mohammad, A. M.; Ghulam, M. R.; Aijaz, A. D. J. Colloid Interface Sci. 2011, 355, 140.

[27] Li, K.; Li, X. M.; Ji, N. Y.; Wang, B. G. Bioorg. Med. Chem. 2007, $15,6627$.

[28] Om, P. S.; Tej, K. B. Food Chem. 2009, 113, 1202. 